\title{
Generation of radial electric field in the process of full reconnection by kinetic kink mode
}

\author{
Taro Matsumoto ${ }^{1}$, Shinji Tokuda ${ }^{1}$, Yasuaki Kishimoto ${ }^{1}$, and Hiroshi Naitou ${ }^{2}$ \\ ${ }^{1}$ Naka Fusion Research Establishment, Japan Atomic Energy Research Institute, 801-1 Mukouyama, Naka 311-0193, Japan \\ ${ }^{2}$ Department of Electrical and Electronic Engineering, Yamaguchi University, 2-16-1 Tokiwadai, Ube 755-8611, Japan
}

(Received June 28, 2000; Revised March 21, 2001; Accepted March 21, 2001)

\begin{abstract}
Effects of density gradient on the kinetic $m=1(m / n=1 / 1)$ internal kink mode in a cylindrical tokamak plasma are studied by the gyro-kinetic particle simulations. When the density gradient is not large enough to change the full reconnection process, the phenomena after the full reconnection, such as the plasma flow and the secondary reconnection, are changed considerably due to the self-generated radial electric field, i.e. the $m / n=0 / 0$ mode. The growing mechanism is explained by the difference of $\mathbf{E} \times \mathbf{B}$ drift motion between ions and electrons, which is caused by the fast parallel motion of electron.
\end{abstract}

\section{Introduction}

In fusion plasma research, the tokamak type device has a great advantage for a candidate of commercial fusion reactor. However, there are still several physical problems to be clarified until the construction and operation of tokamak reactor. Then, it is necessary to clarify this problem experimentally in support of theoretical and numerical results.

In toroidally confined plasmas, magnetohydrodynamics (MHD) instabilities can be driven at the magnetic surfaces where the safety factor $q$ is rational. Here, the quantity $q$ is defined as the limit of the pitch of the magnetic field line for an infinite number of circuits around the torus. In tokamak plasma with $q<1$ at the magnetic axis, an MHD mode with $m / n=1 / 1$ becomes unstable. Here $m$ and $n$ are the poloidal and the toroidal (longitudinal) mode numbers, respectively. The $m=1$ mode has attracted much interest, since it is accompanied by fast magnetic reconnection and triggers a subsequent internal collapse, and this process would be repeated (sawtooth oscillations) in a steady-state operation.

In the early period of plasma research, the internal collapse by the $m=1$ mode has been explained with the resistive MHD model (Kadomtsev, 1975). However, as the plasma temperature is getting higher by the understanding of plasma properties and the development of additional heating devices, the behavior of the $m=1$ mode in many tokamak experiments (Campbell et al., 1986; West et al., 1987), in particular, the magnetic reconnection time and the behavior of the safety factor after the collapse, are not well explained by the resistive MHD model, suggesting the importance of some other effects.

It is also found that the $m=1$ mode can be influenced by kinetic effects of the plasma, such as the electron inertia, density and temperature gradients, finite ion Larmor radius

Copy right $(\mathrm{C}$ The Society of Geomagnetism and Earth, Planetary and Space Sciences (SGEPSS); The Seismological Society of Japan; The Volcanological Society of Japan; The Geodetic Society of Japan; The Japanese Society for Planetary Sciences. and high energy trapped ions. Because the kinetic effects are crucial in the linear and nonlinear development of the $m=1$ mode, a model including the kinetic effects is required to simulate the collisionless nature of the mode.

Wesson pointed out the importance of electron inertia to explain the fast collisionless reconnection (Wesson, 1990). In his theory, the width of the current layer is of the order of the collisionless electron skin depth. Naitou et al. performed a nonlinear gyro-kinetic particle simulation of the $m=1$ mode in a cylindrical geometry with a uniform plasma pressure (Naitou et al., 1995, 1996). They showed that the magnetic reconnection is triggered by the electron inertia in a much faster time scale than that caused by the resistivity, and the magnetic configuration with $q_{\text {min }}<1$ can be reconstructed after the full reconnection. This two step model was firstly proposed with the two-fluid MHD model (Biskamp and Drake, 1994). In the first step, the full reconnection process due to the electron inertia is completed, and the $m=1$ mode of the electrostatic potential retains after the full reconnection for lack of Ohmic dissipation. In the second step, a secondary reconnection is observed due to the current reconcentration which is caused by the $m=1 \mathbf{E} \times \mathbf{B}$ motion.

In this paper, in order to study effects of density gradient on the kinetic $m=1$ mode, we extend the previous gyro-kinetic simulation to the non-uniform plasma with a density gradient. In Section 2, we briefly refer to our simulation model, and describe the model configuration and parameters. In Section 3 , the simulation results with a non-uniform density profile are shown and are compared with those obtained with the uniform density profile. The diamagnetic effect and the density gradient effect are shown in the full reconnection process. The mechanism of the charge separation is explained by the fast parallel motion of electrons. In Section 4, the nonlinear kinetic effects due to the density gradient are also shown. The energy history is also explained with the above results. Finally, a summary is given in Section 5. 
(a)

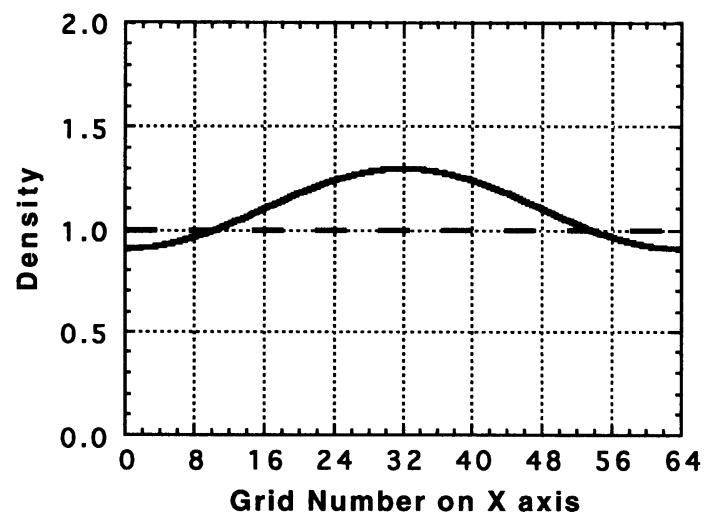

(b)

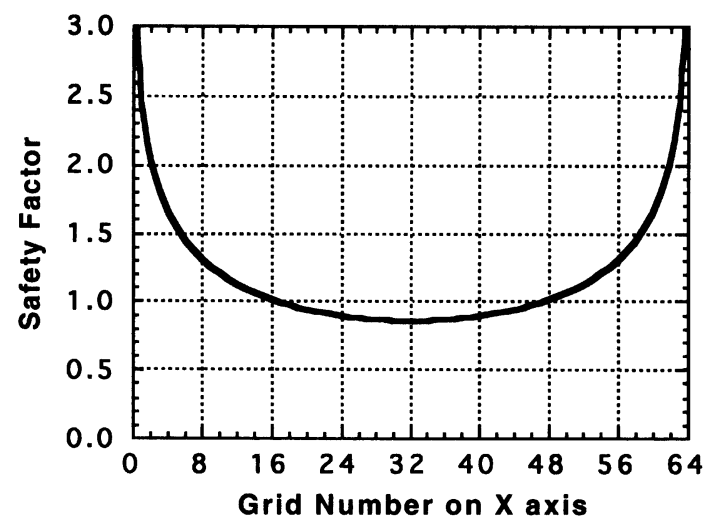

Fig. 1. The equilibrium profiles of (a) the safety factor and (b) the particle density on the $y=32$ line. The solid line and the dashed line indicate the uniform profile and the non-uniform profile, respectively.

\section{Simulation Model}

In our study, we employ a gyro-kinetic model (Lee, 1983) to simulate the $m / n=1 / 1$ mode, because it is efficient compared with the full kinetic model from a viewpoint of the computation. In the gyro-kinetic model, the characteristic time scale is larger than the ion cyclotron period. Hence, the time step can be chosen much larger than the standard particle simulation model in which time step is of the order of electron plasma period.

We adopt a $\delta f$ method (Parker and Lee, 1993) to reduce the statistical noise drastically. In the $\delta f$ method the total distribution function $f_{\sigma}$ is decomposed into equilibrium part $f_{0 \sigma}$ and the perturbed part $\delta f_{\sigma}$;

$$
f_{\sigma}\left(\vec{x}, p_{z}, t\right)=f_{0 \sigma}\left(\vec{x}, p_{z}\right)+\delta f_{\sigma}\left(\vec{x}, p_{z}, t\right),
$$

where $\sigma$ denotes the particle species (ion and electron), $p_{z}$ $\left(=v_{z}+\left(q_{\sigma} / m_{\sigma}\right) A_{z}\right)$ is the longitudinal generalized momentum, $A_{z}$ is the longitudinal vector potential, $q_{\sigma}$ is the electric charge of a particle, and $m_{\sigma}$ is the mass of a particle.

The dynamics of the $\delta f$ are computed in a three dimensional rectangular box with the Cartesian coordinate $(x, y, z)$. Here, a cylindrical plasma configuration is chosen because the toroidal effect is not important for the analysis of the marginal stability problem such as $m=1$ mode. A periodic boundary condition is adopted in the $z$ direction, and a perfect conducting wall is imposed on the $x-y$ boundary surfaces. We choose the initial value of the safety factor at the center $q(0)$ to be equal to $q(0)=0.85$ in the simulation, hence $q$ is unity near the half plasma radius. We assume a uniform temperature profile. The density profile is given by

$$
n_{0}(x, y)=\overline{n_{0}} \frac{\alpha \sin ^{2}\left(\frac{\pi x}{L_{x}}\right) \sin ^{2}\left(\frac{\pi y}{L_{y}}\right)+1}{\frac{1}{4} \alpha+1},
$$

where the density gradient becomes maximum around $q=1$ surface. The factor $\alpha$ is a parameter to control the density gradient, and $\alpha=0$ corresponds to the uniform density case. For the case of the non-uniform density profile, we chose $\alpha=0.44$ in Eq. (2), so that the diamagnetic frequency $\omega_{*} \sim 0.208\left(V_{A} / L_{Z}\right)$ near the $q=1$ surface. The initial (equilibrium) density $n_{0}$ and safety factor profiles are shown in Fig. 1. In the non-uniform density case, the profile of the electron drift velocity $v_{z 0 e}$ is chosen to keep the current density and safety factor profiles in the equilibrium part of the Ampere's law.

In order to include the effect of density gradient in the formulation of the gyro-kinetic equations, we modify the perturbation part of Ampere's law and the time evolution of the weight function (Naitou et al., 1995). First, by considering the contribution of density gradient to the longitudinal vector potential, the perturbation part of Ampere's law is written as

$$
\begin{aligned}
\nabla_{\perp}^{2} \delta A_{z} & -\sum_{\sigma}\left(\frac{\omega_{p \sigma}}{c}\right)^{2} \delta A_{z} \\
= & -\mu_{0} \sum_{\sigma} q_{\sigma}\left(\sum_{j} p_{z j}^{\sigma} w_{j}^{\sigma} S\left[\vec{x}-\vec{x}_{j}^{\sigma}\right]\right) \\
& +\sum_{\sigma}\left(\frac{\omega_{p \sigma}}{c}\right)^{2} A_{z} \frac{1}{n_{0}}\left(\sum_{j} p_{z j}^{\sigma} S\left[\vec{x}-\vec{x}_{j}^{\sigma}\right]\right) \\
& +\sum_{\sigma}\left(\frac{\omega_{p \sigma}}{c}\right)^{2} \frac{n_{0}-\bar{n}_{0}}{\bar{n}_{0}} \delta A_{z},
\end{aligned}
$$

where $\nabla_{\perp}^{2}$ represents a Laplacian perpendicular to the $z$ direction, $\bar{n}_{0}$ is the averaged density in the whole region, $\omega_{p \sigma}$ is a plasma frequency of species $\sigma, c$ is the light velocity in vacuum, $\mu_{0}$ is the vacuum permeability of free space, and $S$ is a particle shape function. The last term represents the effects of non-uniformity of plasma. Next, the time evolution of the weight function of a marker particle $w_{j}^{\sigma}$ is determined by the following equation:

$$
\begin{aligned}
\frac{d w_{j}^{\sigma}}{d t}= & -\left(\frac{f_{0 \sigma}}{g_{0 \sigma}}-w_{j}^{\sigma}\right)\left\{\left[\frac{d x_{j}^{\sigma}}{d t}\left(\frac{q_{\sigma}}{m_{\sigma}} \frac{\partial A_{z 0}}{\partial x}+\frac{\partial v_{z 0 \sigma}}{\partial x}\right)\right.\right. \\
& \left.+\frac{d y_{j}^{\sigma}}{d t}\left(\frac{q_{\sigma}}{m_{\sigma}} \frac{\partial A_{z 0}}{\partial y}+\frac{\partial v_{z 0 \sigma}}{\partial y}\right)\right] \\
& \times \frac{p_{z j}^{\sigma}-\left(q_{\sigma} / m_{\sigma}\right) A_{z 0}-v_{z 0 \sigma}}{2 v_{t \sigma}^{2}} \\
& +\frac{d x_{j}^{\sigma}}{d t} \frac{\partial n_{0} / \partial x}{n_{0}}+\frac{d y_{j}^{\sigma}}{d t} \frac{\partial n_{0} / \partial y}{n_{0}}
\end{aligned}
$$


Table 1. The main parameters used in the simulation. The time and spatial units are normalized with the Alfvén frequency and the ion Larmor radius, respectively.

\begin{tabular}{|l|c||l|c|}
\hline Poloidal mesh width: $\Delta_{\perp}$ & $1\left(\rho_{i}\right)$ & Time step: $\Delta t$ & $2.33 \times 10^{-3}\left(L_{Z} / V_{A}\right)$ \\
System size $\left(L_{X} \times L_{Y} \times L_{Z}\right)$ & $64 \times 64 \times 32$ & Skin depth: $\left(c / \omega_{p e}\right)$ & $4\left(\rho_{i}\right)$ \\
Number of total particles & $4,194,304 \times 2$ & Toroidal mode numbers: $n$ & $-4 \sim+4$ \\
Electron thermal velocity & $0.25\left(V_{A}\right)$ & & \\
\hline
\end{tabular}

$$
\left.+\frac{d p_{z j}^{\sigma}}{d t} \frac{p_{z j}^{\sigma}-\left(q_{\sigma} / m_{\sigma}\right) A_{z 0}-v_{z 0 \sigma}}{2 v_{t \sigma}^{2}}\right\}
$$

Here, $g_{0 \sigma}$ is the distribution function of the marker particles. The second and third terms including the spatial derivatives of the density $n_{0}$ represent the density gradient effect. The gyro-kinetic Poisson's equation is described as

$$
\nabla^{2} \phi+\frac{\omega_{p i}^{2}}{\omega_{c i}^{2}} \nabla_{\perp}^{2} \phi=-\sum_{\sigma} \frac{q_{\sigma}}{\epsilon_{0}}\left(\sum_{j} w_{j}^{\sigma} \delta\left[\vec{x}-\vec{x}_{j}^{\sigma}\right]\right)
$$

where $\omega_{c i}$ is the ion cyclotron frequency. The second term in the left side means the effect of ion polarization. These formulations were described in detail (Matsumoto et al., 1999).

The length and velocity used in this simulation are normalized with the ion Larmor radius $\rho_{i}$ and the Alfvén velocity $L_{Z} / V_{A}$, and the main parameters are listed on Table 1. In the present simulation, we choose the skin depth $\left(\delta_{e}=c / \omega_{p e}\right)$ as $\delta_{e}=4 \rho_{i}$, and the grid size in the $z$-direction as 1000 times larger than that in $x$ or $y$-direction.

\section{Internal Collapse}

\subsection{Diamagnetic effect}

It is well known that the behavior of the $m / n=1 / 1$ mode is affected by the pressure gradient due to the diamagnetic effect. The linear growth rate of the $1 / 1$ mode is restricted with the dependency of a square of the diamagnetic frequency $\omega_{*}$, as been evaluated by the resistive and kinetic models (Zakharov and Rogers, 1992).

In our simulation model, the diamagnetic effect is induced only by the density gradient, since the temperature profile is assumed to be uniform. Figure 2 shows the linear growth rate of the $n=1$ mode in the presence of the density gradient. The $\omega_{*}$ stabilization is found to have a parabolic dependence, as been expected from the theoretical analysis.

In order to clarify the nonlinear kinetic effect of the density gradient on the sawtooth crash, for the case of the non-uniform density profile, we mainly choose $\omega_{*}=$ $0.196 V_{A} / L_{Z}$, which is much less than the growth rate for the uniform density $\left(\gamma_{0} \sim 0.87 V_{A} / L_{Z}\right)$. Then, the linear growth of the $m=1$ mode is little affected by the density gradient. Therefore, the $\omega_{*}$ stabilization is negligible, however, the nonlinear kinetic effect of the density gradient becomes clear as mentioned in the next section.

\subsection{Full magnetic reconnection}

Next, the simulation results of the non-uniform density case are compared with those obtained for the uniform density case. As a nonlinearly changeable factor, the behavior of the magnetic structure should be paid attention to. For the analysis of the nonlinear variation of magnetic field lines, it is useful to draw the Poincare plots, where the position of a

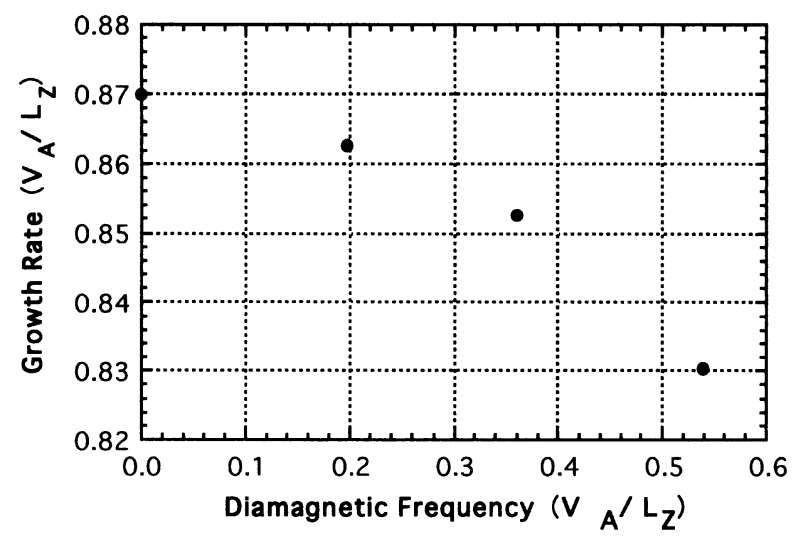

Fig. 2. The dependence of the linear growth rate of the $n=1$ mode on the diamagnetic frequency.

field line on the $z=0$ plane is drawn by a point and the magnetic surface is described by the set of points for a number of circuits around the torus. Figure 3 shows the Poincare plots before and after the internal collapse (the flattening event in the central region) in the simulations with (I) non-uniform and (II) uniform density profiles. As shown in Fig. 3(I-a) before the internal collapse, the core plasma has concentric flux surfaces and the $m=1$ magnetic island has a crescent shape. The core plasma is swept out from the central region due to the $\mathbf{E} \times \mathbf{B}$ motion induced by the $m=1$ mode of the electrostatic potential which grows inside the $q=1$ rational surface. On the other hand, the $m=1$ magnetic island comes into the central region. As a result, the reconnection with topological variation is completed without saturation (the so-called, "full reconnection"), and the magnetic configuration has nested flux surfaces as shown in Fig. 3(I-b). The process of the full reconnection with the density gradient is almost the same as that obtained with the uniform density profile as shown in Fig. 3(II-a,b).

Figures 3(I-c) and (II-c) are the magnetic configurations from which we find the discrepancy between them. For the uniform density case (II-c), a new reconnection process occurs after the full reconnection (the so-called, "secondary reconnection"), while for the non-uniform density case (I-c), the secondary reconnection is unclearly seen.

Figure 4 shows the electrostatic potential energy decomposed in the longitudinal mode numbers $n$ in (I) the nonuniform and (II) the uniform density cases. After the full reconnection is completed $\left[t=34 \sim 35\left(V_{A} / L_{Z}\right)\right.$, near the arrows of (I-b) and (II-b)], the $n=1$ mode is dominant in both cases. Figure 5 shows the electrostatic potential contours at $z=0$, where the plus value means the dominance 

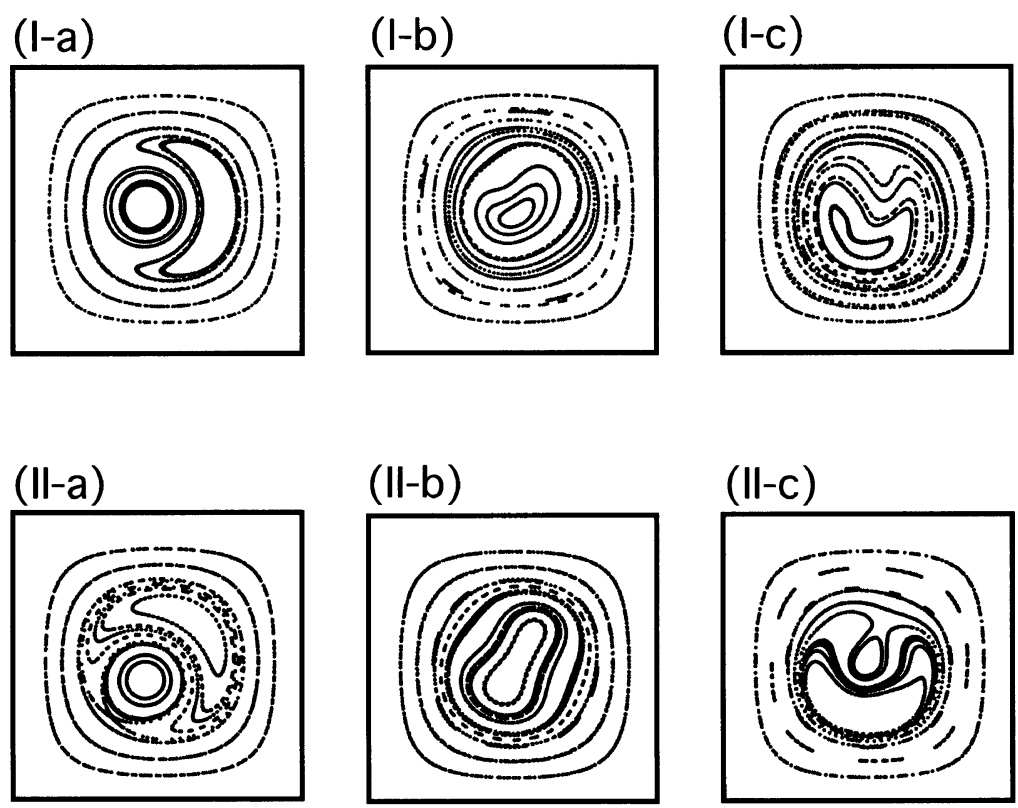

Fig. 3. Poincare Plots of the non-uniform density simulation at (I-a) $t=30.11$, (I-b) $t=34.54$, (I-c) $t=37.34$ and the uniform density simulation at (II-a) $t=29.81$, (II-b) $t=33.79$, (II-c) $t=37.28$. (I-a) and (II-a) show the shifted magnetic surfaces due to the $1 / 1$ mode. (I-b) and (II-b) show the nested surfaces just after the full reconnection. (I-c) and (II-c) show the secondary reconnection process.
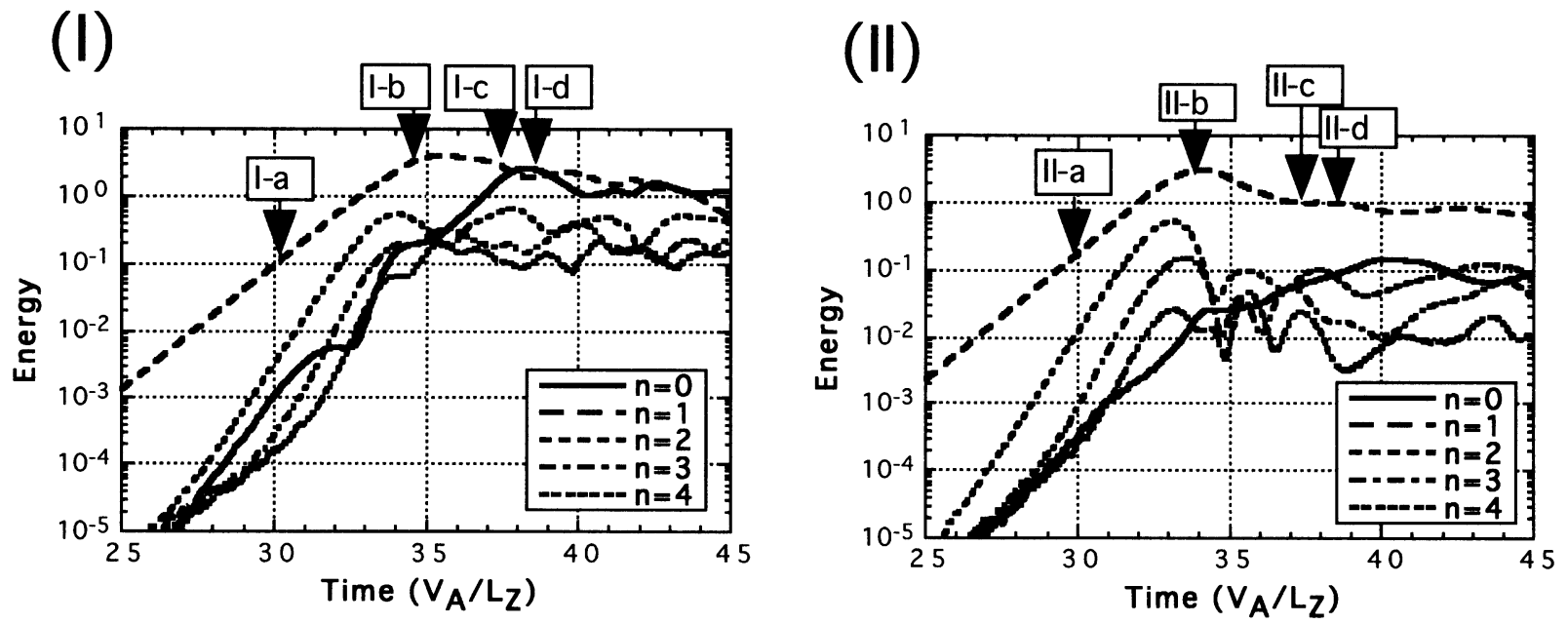

Fig. 4. The time evolution of the electrostatic potential energy decomposed in the longitudinal mode numbers for (I) the non-uniform density simulation and (II) the uniform density simulation.

of ions. In the poloidal direction, the $m=1$ mode is obviously dominant in both cases, as seen in Fig. 5(I-b) and (II-b). Then, the $m / n=1 / 1$ mode is dominant through the full reconnection process.

\subsection{Charge separation}

From the above results, it seems that the behavior of the magnetic configuration and the $1 / 1$ mode is not affected so much by the density gradient during the full reconnection. However, as the core plasma is swept out due to the $\mathbf{E} \times$ B flow, the $0 / 0$ mode of the electrostatic potential grows with negative sign at the central region. Figure 6 shows the difference of the $n=0$ mode energy between the nonuniform and the uniform density cases, which means the charge separation with the increase of the plasma shift until the end of the full reconnection $\left(t \sim 32.5 V_{A} / L_{Z}\right)$.

The elementary process causing the charge separation is explained by the difference of motion between ions and electrons. The parallel velocity of electrons is much faster than that of ions by $\left(m_{i} / m_{e}\right)^{1 / 2}$ if $T_{e} \sim T_{i}$. Then, in the process of $\mathbf{E} \times \mathbf{B}$ motion, the electrons are obedient to the electric field averaged along the magnetic field line, while the ions faithfully submit to the local electric field. As a result, the electron $\mathbf{E} \times \mathbf{B}$ motion delays compared with the ion motion. In the presence of the density gradient, this process can induce the charge separation. Therefore, the radial electric field is closely relevant to the motion of high-density plasma region. 

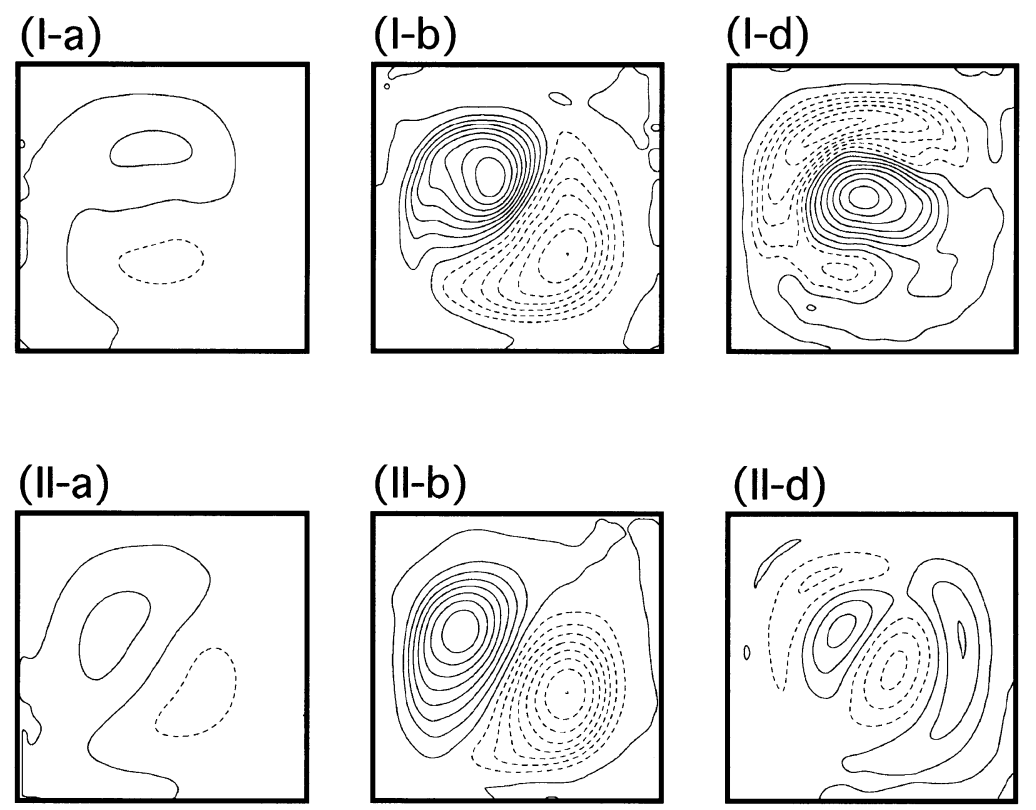

Fig. 5. The contours of the electrostatic potential in the non-uniform density simulation at (I-a) $t=30.11$, (I-b) $t=34.54$, (I-d) $t=38.51$ and the uniform density simulation at (II-a) $t=29.81$, (II-b) $t=33.79$, (II-d) $t=38.45$. The solid and dashed lines indicate the plus and minus contour lines, respectively. (I-b) and (II-b) show the $1 / 1$ mode just after the full reconnection. (I-d) and (II-d) show the secondary reconnection process.

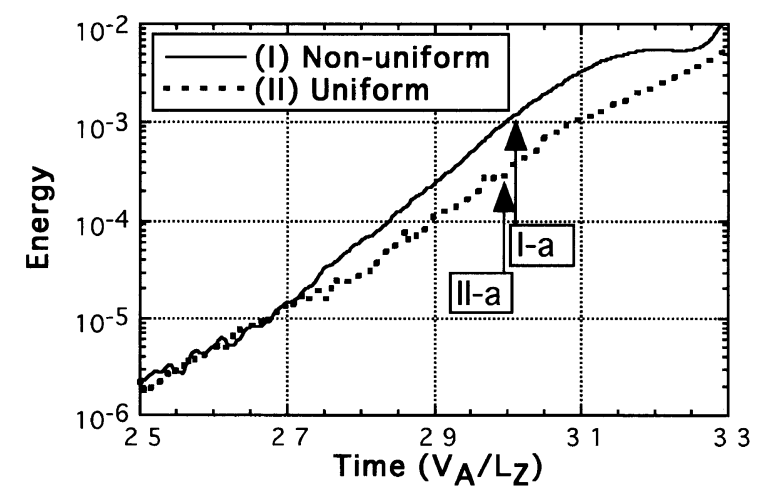

Fig. 6. The time evolution of the $n=0$ electrostatic potential energy with the increase of the plasma shift for (I) the non-uniform and (II) the uniform density cases.

\section{Nonlinear Effects}

\subsection{Nonlinear evolution of radial electric field}

After the full reconnection, on the contrary, the density gradient is found to affect considerably the nonlinear behavior of plasma. In the presence of the density gradient, the $n=0$ mode grows exponentially to be the same level as that of the $n=1$ mode, as shown in Fig. 4 (I) $\left(t=35 \sim 38 V_{A} / L_{Z}\right)$. At the same time, we found that the $m=0$ component of the electrostatic potential, i.e. the radial electric field, grows up as shown in Fig. 5(I-d). Then, it is found that the $0 / 0$ mode grows nonlinearly with positive sign at the central region.

On the other hand, the radial electric field is not observed in the uniform density case. In the poloidal direction, the $m=1$ mode is still dominant, although the electrostatic potential has a reversed structure radially. Then, the $m / n=1 / 1$ mode is dominant at $t=38.45\left(V_{A} / L_{Z}\right)$, as can be seen in Fig. 5(II-d) and Fig. 4(II).
The evolution of the density profile in the nonlinear phase is affected by the $\mathbf{E} \times \mathbf{B}$ rotation due to the self-generated radial electric field. The black region in Fig. 7 represents the high-density region in the non-uniform density case. The high-density region of the initial equilibrium (I-a) is swept out from the central region because of the full reconnection, as shown in Fig. 7(I-b). After the full reconnection, the highdensity plasma rotates in the ion diamagnetic direction, and comes into the central region spirally as shown in Fig. 7(I-c).

The formation of the vortex structure is found to be closely related with the radial electric field. The mechanism of the nonlinear growth of the $0 / 0$ mode and the vortex density is shown prior to this.

\subsection{Physical mechanism}

The $\mathbf{E} \times \mathbf{B}$ motion just after the full reconnection is sustained by the $m=1$ mode, as shown in Fig. 5(I-b). The high dense plasma comes into the central region again by the symmetrical flow induced by the $m=1$ mode. At that moment, the ion comes inside faster than the electron due to the electron delay process mentioned in the previous section. Then, the weak charge separation makes the seed radial electric field, which changes the symmetrical flow pattern into the asymmetrical one, as shown in Fig. 7(I-b).

Once the seed field is generated, the $\mathbf{E} \times \mathbf{B}$ rotation is induced, and more dense plasma enters into the central region. Then, the charge separation is facilitated due to the electron delay. As a result, the radial electric field is self-generated by this positive feedback mechanism. Finally, the growth of the radial field is terminated when the potential energy of the $0 / 0$ mode is comparable to that of the $1 / 1$ mode, because the charge separation is driven by the original symmetrical flow.

\section{Summary}

The density gradient effect is taken into account in a gyrokinetic nonlinear simulation of the kinetic $m=1$ internal 

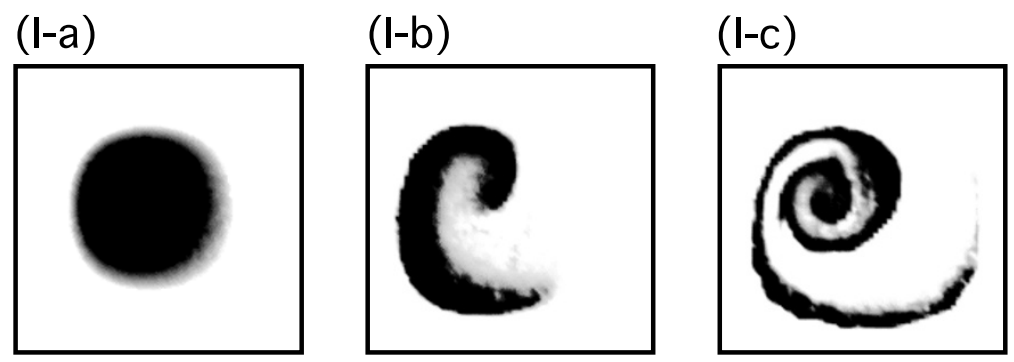

Fig. 7. The high-density regions of electrons in the peaked density simulation at (I-a) $t=30.11$, (I-b) $t=34.54$, and (I-c) $t=37.34$.

kink mode in a cylindrical tokamak plasma. The simulation results of the non-uniform density case are compared with those of the uniform density case. The core plasma is swept out by the $\mathbf{E} \times \mathbf{B}$ motion due to the $m=1$ electric field produced by the unstable kinetic internal kink mode. The process of the full reconnection is completed without saturation, and is almost the same as the one obtained for the uniform density simulation.

It is found that even when $\omega^{*}$ is not large enough to change the linear growth rate of the $1 / 1$ mode, the nonlinear phenomena after the full reconnection are considerably changed due to the self generated radial electric field, i.e. 0/0 mode. This mode is induced by the electron delay process during the $\mathbf{E} \times \mathbf{B}$ motion in the presence of the density gradient. The growing mechanism is explained by the difference of $\mathbf{E} \times \mathbf{B}$ drift motion between ions and electrons, which is caused by the fast parallel motion of electron.

Once the radial electric field is triggered by the symmetrical flow induced by the $1 / 1$ mode, the $0 / 0$ mode grows up to the same level as the 1/1 mode, and drives an $\mathbf{E} \times \mathbf{B}$ plasma rotation in the ion diamagnetic direction. This plasma rotation breaks the symmetrical plasma flow induced by the $1 / 1$ mode. Then, the density profile becomes a vortex structure. It is also found that the formation of the vortex is closely interacted with the growth of the radial electric field, and constitutes a kind of positive feedback mechanism. It should be noted that a radial electric field with positive central charge is experimentally obtained in the JIPP T-IIU tokamak just after the internal collapse of the sawtooth oscillations (Hamada et al., 1996).

For the non-uniform temperature case, the nonlinear evolution of the radial electric field would be enhanced a little, because the parallel electron motion of the central highdensity region increases. In high temperature plasmas, the effect of FLR (Finite Larmor Radius) becomes important. In the case of large FLR compared with electron skin depth, the reduction of the linear growth rate can be expected from the two-fluid theory. The large FLR effect is being analyzed now, and will be shown in the near future.

Acknowledgments. This research is a part of the NEXT (Numerical EXperimental Tokamak) project promoted by JAERI. The authors express their gratitude to Drs. T. Takizuka and M. Azumi for their useful suggestions and encouragement. The authors thank Professor M. Wakatani of Kyoto University and Professor O. Fukumasa of Yamaguchi University for their helpful comments.

\section{References}

Biskamp, D. and J. F. Drake, Dynamics of the sawtooth collapse in tokamak plasmas, Phys. Rev. Lett., 73, 971-974, 1994.

Campbell, D. J., R. D. Jill, C. W. Gowers, et al., Sawtooth activity in Ohmically heated JET plasmas, Nuclear Fusion, 26, 1085, 1986.

Hamada, Y., A. Nishizawa, Y. Kawasumi, et al., Fast potential change during sawteeth in JIPP T-IIU tokamak plasmas, Nuclear Fusion, 36, 515-520, 1996.

Kadomtsev, B. B., Disruptive instability in tokamaks, Sov. J. Plasma Phys., 1, 389-391, 1975 .

Lee, W. W., Nonlinear gyrokinetic equations, Phys. Fluids, 26, 556-562, 1983.

Matsumoto, T., S. Tokuda, Y. Kishimoto, et al., Gyro-kinetic particle simulation of $m=1$ internal kink mode in the process of density gradient, $J$. Plasma and Fusion Research, 75, 1188-1194, 1999.

Naitou, H., K. Tsuda, W. W. Lee, and R. D. Sydora, Gyrokinetic simulation of internal kink modes, Phys. Plasma, 2, 4257-4268, 1995.

Naitou, H., T. Sonoda, S. Tokuda, and V. K. Decyk, Parallelization of gyrokinetic particle code and its application to internal kink mode simulation, J. Plasma and Fusion Research, 72, 259-269, 1996.

Parker, S. E. and W. W. Lee, A fully nonlinear characteristic method for gyrokinetic simulation, Phys. Fluids, B5, 77-86, 1993.

Wesson, J. A., Sawtooth reconnection, Nuclear Fusion, 30, 2545-2549, 1990

West, W. P., D. M. Thomas, and J. S. de Grassie, Measurement of the rotational transform at the axis of a tokamak, Phys. Rev. Lett., 58, 27582761, 1987.

Zakharov, L. and B. Rogers, Two-fluid magnetohydrodynamic description of the internal kink mode in tokamaks, Phys. Fluids, B4, 3285-3301, 1992.

T. Matsumoto (e-mail: tmatsumo@naka.jaeri.go.jp), S. Tokuda, Y. Kishimoto, and H. Naitou 